\title{
IMAGES AND LEVEL SETS OF ADDITIVE RANDOM WALKS*
}

\author{
PROC. CONF. ON HIGH DIM. PROB. (Seattle 1999; to appear) \\ Davar Khoshnevisan \\ Yimin Xiao \\ University of Utah \\ University of Utah \\ and \\ Microsoft Corporation
}

August 10, 2001

\section{Introduction}

We define $\mathbb{N}_{0}=\{0,1, \ldots\}$ and say that a stochastic process $X=\{X(n)$; $\left.n \in \mathbb{N}_{0}^{N}\right\}$ is an $N$-parameter, $\mathbb{Z}^{d}$-valued, additive random walk, if there are $N$ independent random walks $X_{1}, \ldots, X_{N}$ on $\mathbb{Z}^{d}$, such that

$$
X(n)=X_{1}\left(n_{1}\right)+\cdots+X_{N}\left(n_{N}\right), \quad n \in \mathbb{N}_{0}^{N} .
$$

Here, $n_{j}$ denotes the $j$ th coordinate of $n \in \mathbb{N}_{0}^{N}$ and we are following the standard convention of starting our (ordinary) random walks at the origin. That is, $X_{j}(0)=0$ for all $j=1, \ldots, N$. From now on, $N$ will always denote the temporal dimension and $d$ the spatial one, in accordance with the conventional language of stochastic processes.

The continuous time analogue of such random walks plays an important rôle in the analysis of Lévy sheets and, in particular, Brownian sheet; cf. Ref.'s $[4,5,6,7,12,13,14,17,18]$ to cite only some of the references. These additive random walks also occur naturally in the theory of intersections of Lévy processes; cf. $[9,10,16]$ and in the applications of probability theory in constructing exceptional sets and in harmonic analysis; cf. [11] and its extensive list of references.

In this article, we study the distributional properties of the the level sets of $X$ by analyzing the 'size' of the random image $X(E)$ where $E \subset \mathbb{N}_{0}^{N}$ is

\footnotetext{
${ }^{*}$ Research partially supported by a grant from the NSF and a grant from NATO
} 
any nonrandom set. We will also show how such general results characterize 'recurrence' and 'transience' of $X$. As such, the results of the present paper are closely related to the companion paper [15]. In order to present the main result of this paper, we define the function $\Phi: \mathbb{N}_{0}^{N} \times \mathbb{N}_{0}^{N} \mapsto[0,1]$ as

$$
\Phi(n, m)=\mathbb{P}\{X(n)=X(m)\}, \quad n, m \in \mathbb{N}_{0}^{N},
$$

and refer to this $\Phi$ as the gauge function of the additive random walk $X$. For all probability measures $\mu$ on $\mathbb{N}_{0}^{N}$, we can define the $\Phi$-energy of $\mu$ as

$$
\mathcal{E}_{\Phi}(\mu)=\iint \Phi(x, y) \mu(d x) \mu(d y) .
$$

Then, the $\Phi$-capacity $\mathcal{C}_{\Phi}(E)$ of any $E \subset \mathbb{N}_{0}^{N}$ can be defined by the principle of minimum energy:

$$
\mathcal{C}_{\Phi}(E)=\left[\inf \left\{\mathcal{E}_{\Phi}(\mu): \mu \in \mathcal{P}(E) \text { and } \mathcal{E}_{\Phi}(\mu)>0\right\}\right]^{-1},
$$

where $\mathcal{P}(E)$ denotes the collection of all probability measures on $E$, inf $\varnothing=$ $+\infty$ and $1 / \infty=0$.

The first main result of this article is the following theorem that estimates the size of the image of $E$ under the random map $X$.

Theorem 1.1. Let $X$ be an $N$-parameter, d-dimensional additive random walk with gauge function $\Phi$. For all $E \subset \mathbb{N}_{0}^{N}$,

$$
\mathcal{C}_{\Phi}(E) \leqslant \mathbb{E}\{\# X(E)\} \leqslant 16^{N} \mathcal{C}_{\Phi}(E),
$$

where $\# X(E)$ denotes the cardinality of $X(E)$.

Remark 1.1. When $N=1$ and $X$ is an ordinary random walk on $\mathbb{Z}^{d}$, constant 16 in (1.4) can be replaced by 2 . cf. Remark 4.1 in $\S 4$.

Next, we illustrate the power of such a general estimate by studying the level sets of a large class of additive random walks.

We say that our additive random walk $X$ is symmetric, if for each $n \in \mathbb{N}_{0}^{N}$, $-X(n)$ has the distribution as $X(n)$. Clearly the symmetry of the additive random walk $X$ implies that for each $j=1, \ldots, N$, the classical random walk $X_{j}$ is also symmetric. 
Note that in case $X$ is symmetric, $\Phi(n, m)$ is a function of $n-m \in \mathbb{Z}^{N}$. That is, we can define $\Psi: \mathbb{Z}^{N} \mapsto[0,1]$ in a unique and well-defined manner by

$$
\Psi(n-m)=\Phi(n, m),
$$

where $n, m \in \mathbb{N}_{0}^{N}$. Let $\bar{n}$ denote the point in $\mathbb{N}_{0}^{N}$ whose $j$ th coordinate is $\left|n_{j}\right|$. Then, we point out that for all $n, m \in \mathbb{N}_{0}^{N}$,

$$
\Phi(n, m)=\Psi(n-m)=\mathbb{P}\{X(\overline{n-m})=0\} .
$$

We also write $\mathcal{C}_{\Psi}(E)$ and $\mathcal{E}_{\Psi}(\mu)$ for $\mathcal{C}_{\Phi}(E)$ and $\mathcal{E}_{\Phi}(\mu)$, in the symmetric case, and also refer to $\Psi$ as the (symmetrized) gauge function of $X$.

In order to describe a natural partial order on $\mathbb{Z}^{N}$, we define the partial order ' $\preccurlyeq$ ' on $\mathbb{Z}^{N}$ by: for all $m, n \in \mathbb{Z}^{N}, m \preccurlyeq n$ if and only if $m_{j} \leqslant n_{j}$ for all $j=1, \ldots, N$. This partial order induces a minimum (maximum, resp.) operator $\curlywedge\left(\curlyvee\right.$, resp.): for all $m, n \in \mathbb{Z}^{N}, m \curlywedge n(m \curlyvee n$, resp.) denotes the point in $\mathbb{Z}^{N}$ whose $j$ th coordinate is $\min \left\{m_{j}, n_{j}\right\}\left(\max \left\{n_{j}, m_{j}\right\}\right.$, resp.) for all $j=1, \ldots, N$.

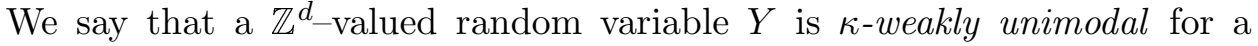
positive constant $\kappa \geqslant 1$, when

$$
\sup _{a \in \mathbb{Z}^{d}} \mathbb{P}\{Y=a\} \leqslant \kappa \mathbb{P}\{Y=0\} .
$$

Throughout the remainder of the Introduction, we will assume the existence of a fixed constant $\kappa \geqslant 1$ such that whenever $m, n \in \mathbb{N}_{0}^{N}$ with $\mathbb{P}\{X(m)=$ $0, X(n)=0\}>0, X(m \curlywedge n)$ is $\kappa$-weakly unimodal. When this is so, we say that the $N$-parameter random walk $X:=\left\{X(n) ; n \in \mathbb{N}_{0}^{N}\right\}$ is weakly unimodal. We observe that the above definition of weak unimodality of $X$ implies that whenever $m \in \mathbb{N}_{0}^{N}$ with $\mathbb{P}\{X(m)=0\}>0, X(m)$ is weakly unimodal.

For all $E \subset \mathbb{N}_{0}^{N}$, we write $\delta_{E}\left(\Delta_{E}\right.$, resp.) for any choice of $n \in \mathbb{N}_{0}^{N}$ with

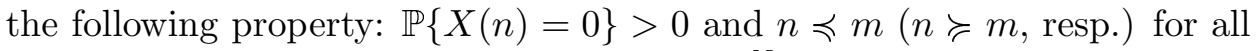
$m \in E$. We note that $\delta_{E}$ always exists in $\mathbb{N}_{0}^{N}$, and $\Delta_{E}$ also exists as long as $E$ is bounded and $X$ symmetric. If $\mathbb{P}\{X(n)=0\}=0$ for all $n \succcurlyeq E$ (i.e. $n \succcurlyeq m$ for all $m \in E$ ) or $E$ is infinite, we define $\Delta_{E}=\infty$. Of course, it is not necessarily true that $\delta_{E}$ or $\Delta_{E}$ are themselves in $E$. 
Theorem 1.2. Let $X$ be a symmetric, weakly unimodal, $N$-parameter additive random walk with symmetrized gauge function $\Psi$. Then, for all $E \subset \mathbb{N}_{0}^{N}$,

$$
\begin{aligned}
\frac{\Psi^{2}\left(\Delta_{E}\right)}{\kappa^{3} \Psi\left(\delta_{E}\right)} \cdot \mathcal{C}_{\Psi}(E) \leqslant & \mathbb{P}\{0 \in X(E)\} \\
& \leqslant \sup _{a \in \mathbb{Z}^{d}} \mathbb{P}\{a \in X(E)\} \\
& \leqslant \kappa 16^{N} \Psi\left(\delta_{E}\right) \cdot \mathcal{C}_{\Psi}(E),
\end{aligned}
$$

where $\Psi(\infty)=0$ and $0 / 0=0$.

Amongst other things, the above estimates the probability that the zero set of $X$ intersects a given set $E$ and this estimate is essentially sharp, as long as $\Psi\left(\delta_{E}\right)$ and $\Psi\left(\Delta_{E}\right)$ are of the same order of magnitude.

Next, let us denote the level set of $X$ (at 0 ) by $\mathcal{L}$. That is,

$$
\mathcal{L}=\left\{n \in \mathbb{N}_{0}^{N}: X(n)=0\right\}
$$

When $N=1$, a classical problem of probability has been to establish when $\mathcal{L}$ is an infinite set. Equivalently, the question is: "when is 0 recurrent?" We shall continue using this notation for all $N \geqslant 1$. Our next theorem gives a necessary and sufficient condition for 0 to be recurrent for the additive random walk $X$. Needless to say, one needs to better understand the notion of recurrence in order to work in the multiparameter setting. For instance, the reader is reminded that notions of 'stopping times', 'first time to ...', etc. no longer even make sense. As such, other ideas are needed to prove the following.

Theorem 1.3. Suppose $X$ is a symmetric, weakly unimodal, $N$-parameter additive random walk on $\mathbb{Z}^{d}$ and $X(0)=0$, the following are equivalent:

1. 0 is recurrent with positive probability;

2. 0 is recurrent with probability one; and

3. $\sum_{n \in \mathbb{N}_{0}^{N}} \mathbb{P}\{X(n)=0\}=\infty$.

It follows from Theorem 1.3 that if $\sum_{n \in \mathbb{N}_{0}^{N}} \mathbb{P}\{X(n)=0\}=\infty$, then $\lim _{n \rightarrow \infty} \#\left(\mathcal{L} \cap[0, n]^{N}\right)=\infty$, almost surely, where \# denotes cardinality. Due to its close connections to the various fractal dimensions of $\mathcal{L}$, the rate 
at which $\#\left(\mathcal{L} \cap[0, n]^{N}\right) \rightarrow \infty$ is a matter of some importance; see $[1,2]$ for results about discrete fractals determined by classical random walks. Here, we define the following two indices:

$$
\begin{aligned}
& \bar{\gamma}=\inf \left\{\beta>0: \limsup _{n \rightarrow \infty} n^{-\beta} \sum_{k \in[0, n]^{N}} \Psi(k)<\infty\right\}, \text { and } \\
& \underline{\gamma}=\sup \left\{\beta>0: \liminf _{n \rightarrow \infty} n^{-\beta} \sum_{k \in[0, n]^{N}} \Psi(k)>0\right\} .
\end{aligned}
$$

It is easy to verify that $0 \leqslant \underline{\gamma} \leqslant \bar{\gamma} \leqslant N$. Whenever $\underline{\gamma}=\bar{\gamma}$, we write their common value as $\gamma$. Later on, we will use the following elementary fact: for any $\varepsilon>0$, there exists a positive and finite constant $K$ such that

$$
K^{-1} n \underline{\gamma}^{-\varepsilon} \leqslant \sum_{k \in[0, n]^{N}} \Psi(k) \leqslant K n^{\bar{\gamma}+\varepsilon}
$$

for all $n$ large enough.

In the following, we denote $\mathcal{L}(n)=\#\left(\mathcal{L} \cap[0, n]^{N}\right)$.

Theorem 1.4. Suppose $X$ is a symmetric, weakly unimodal, $N$-parameter additive random walk on $\mathbb{Z}^{d}$. Then, almost surely,

$$
\underline{\gamma} \leqslant \limsup _{n \rightarrow \infty} \frac{\log \mathcal{L}(n)}{\log n} \leqslant \bar{\gamma}
$$

In particular, under the conditions of Theorem 1.4, whenever $\underline{\gamma}=\bar{\gamma}$,

$$
\limsup _{n \rightarrow \infty} \frac{\log \mathcal{L}(n)}{\log n}=\gamma
$$

almost surely.

The outline of the remainder of this article is as follows. In Section 2, we establish a series of Markov properties for additive random walks; this is an important step in our proof of Theorem 1.1. In Section 3, we prove some technical lemmas and give some examples of weakly unimodal additive random walks. Theorems 1.1 and 1.2 are proved in Sections 4 Section 5, respectively. Finally, in Section 6, we prove Theorems 1.3 and 1.4. 


\section{Markov Properties}

The additive random walks of $\S 1$ satisfy $2^{N}$ 'Markov properties' that are immediately relevant to the results of this paper. However, to get these Markov properties. we will need to use non-probability measures. To do this concisely, we work on the canonical probability space $(\Omega, \mathcal{F})$, where $\Omega=\left(\mathbb{Z}^{d}\right)^{\mathbb{N}_{0}} \oplus \cdots \oplus\left(\mathbb{Z}^{d}\right)^{\mathbb{N}_{0}}$ is the collection of all functions $\omega: \mathbb{N}_{0}^{N} \mapsto \mathbb{Z}^{d}$ of form $\omega(n)=\omega_{1}\left(n_{1}\right)+\cdots+\omega_{N}\left(n_{N}\right)$, where $\omega_{j} \in\left(\mathbb{Z}^{d}\right)^{\mathbb{N}_{0}}$. Moreover, $\mathcal{F}$ denotes the Borel field based on the product topology. The processes $X, X_{1}, \ldots, X_{N}$ are then described in the usual way as follows: for all $\omega \in \Omega$, all $n \in \mathbb{N}_{0}^{N}$ and all $j=1, \ldots, N, X(n)(\omega)=\omega(n), X_{j}\left(n_{j}\right)=\omega_{j}\left(n_{j}\right)$. Let $\mathbb{P}$ denote the probability measure - derived from Kolmogorov's existence theorem-that makes this coordinatewise definition of $X$ a version of the additive random walk we need, and define for all $A \in \mathcal{F}$,

$$
\mathbb{Q}(A)=\sum_{a \in \mathbb{Z}^{d}} \mathbb{P}\{\omega \in \Omega: \omega+a \in A\}
$$

where, as usual, $(\omega+a)(n)=\omega(n)+a\left(n \in \mathbb{N}_{0}^{N}\right)$. The measure $\mathbb{Q}$ is $\sigma$-finite but not finite. In fact, for all $n \in \mathbb{N}_{0}^{N}$ and $b \in \mathbb{Z}^{d}, \mathbb{Q}\{X(n)=b\}=1$. We denote by $\mathbb{E}_{\mathbb{Q}}$ the abstract integral (or 'expectation') with respect to the infinite measure $\mathbb{Q}$. That is, for all $Z: \Omega \mapsto \mathbb{R}_{+}, \mathbb{E}_{\mathbb{Q}}\{Z\}=\int_{\Omega} Z(\omega) \mathbb{Q}(d \omega)$, and this uniquely defines $\mathbb{E}_{\mathbb{Q}}\{Z\}$, at least whenever $\mathbb{E}_{\mathbb{Q}}\{|Z|\}<\infty$. There are $2^{N}$ standard partial orders on $\mathbb{N}_{0}^{N}$, all of which we will need. A good way to keep track of them is as follows: let $\Pi$ denote the collection of all subsets of $\{1, \ldots, N\}$. For all $A \in \Pi$, we then define a partial order $\preccurlyeq(A)$ on $\mathbb{N}_{0}^{N}$ by declaring $n \preccurlyeq(A) m$ whenever for all $j \in A, n_{j} \leqslant m_{j}$ and for all $j \notin A, n_{j} \geqslant m_{j}$. We will not distinguish between $n \preccurlyeq(A) m$ and $m \succcurlyeq_{(A)} n$. It is important to notice that the partial order " $\preccurlyeq(\{1, \ldots, N\})$ " is the same as "ঝ" of the Introduction.

For each $A \in \Pi$ and for all $n \in \mathbb{N}_{0}^{N}$, we define $\mathcal{F}^{A}(n)$ to be the $\sigma$-field generated by the collection $\{X(m) ; m \preccurlyeq(A) n\}$. Clearly, $\mathcal{F}^{A}$ is a filtration in the partial order $\preccurlyeq(A)$. That is, whenever $n \preccurlyeq(A) m, \mathcal{F}^{A}(n) \subset \mathcal{F}^{A}(m)$. We will also need a kind of 'reversed filtration' $\mathcal{G}^{A}$ for each $A \in \Pi$ : for all $n \in \mathbb{N}_{0}^{N}, \mathcal{G}^{A}(n)$ denotes the $\sigma$-field generated by $\left\{X(m) ; m \succcurlyeq_{(A)} n\right\}$.

Proposition 2.1 (Markov Properties). For every $A \in \Pi$ and all $n \in \mathbb{N}_{0}^{N}$, $\mathcal{G}^{A}(n)$ is conditionally independent of $\mathcal{F}^{A}(n)$, given $X(n)$, under the measure $\mathbb{Q}$.

Remark 2.1. When $N \geq 2$, this is not true under $\mathbb{P}$. 
In order to explain Proposition 2.1, let us fix $A \in \Pi$ and suppose $p, q \in \mathbb{N}_{0}$ and $k_{-p}, \ldots, k_{q} \in \mathbb{N}_{0}^{N}$ satisfy the following: for all $1 \leqslant i \leqslant p$ and $1 \leqslant j \leqslant q$, $k_{-i} \preccurlyeq_{(A)} k_{0} \preccurlyeq_{(A)} k_{j}$. Then, Proposition 2.1 states that for all $f_{\ell}: \mathbb{Z}^{d} \mapsto \mathbb{R}_{+}$ $(-p \leqslant \ell \leqslant q)$,

$$
\begin{aligned}
\mathbb{E}_{\mathbb{Q}}\left\{\prod_{\ell=-p}^{q} f_{\ell}\left(X\left(k_{\ell}\right)\right) \mid X\left(k_{0}\right)\right\} \\
=f_{0}\left(X\left(k_{0}\right)\right) \times \mathbb{E}_{\mathbb{Q}}\left\{\prod_{\ell=-p}^{-1} f_{\ell}\left(X\left(k_{\ell}\right)\right) \mid X\left(k_{0}\right)\right\} \\
\times \mathbb{E}_{\mathbb{Q}}\left\{\prod_{\ell=1}^{q} f_{\ell}\left(X\left(k_{\ell}\right)\right) \mid X\left(k_{0}\right)\right\},
\end{aligned}
$$

$\mathbb{Q}$-almost surely.

Proof. Using the notation used in Eq. (2.2),

$$
\begin{aligned}
& \mathbb{E}_{\mathbb{Q}}\left\{\prod_{\ell=-p}^{q} f_{\ell}\left(X\left(k_{\ell}\right)\right)\right\} \\
= & \sum_{a \in \mathbb{Z}^{d}} \mathbb{E}\left\{\prod_{\ell=-p}^{q} f_{\ell}\left(X\left(k_{\ell}\right)+a\right)\right\} \\
= & \left.\sum_{a \in \mathbb{Z}^{d}} f_{0}(a) \cdot \mathbb{E}\left\{\prod_{\substack{-p \leqslant \ell \leqslant q \\
\ell \neq 0}} f_{\ell}\left(X\left(k_{\ell}\right)-X\left(k_{0}\right)+a\right)\right)\right\},
\end{aligned}
$$

as long as any the latter sum is finite. We now use the easily verifiable fact that the following collections of random variables are independent from one another:

(i) $\left\{X\left(k_{-p}\right)-X\left(k_{0}\right), \ldots, X\left(k_{-1}\right)-X\left(k_{0}\right)\right\}$; and

(ii) $\left\{X\left(k_{1}\right)-X\left(k_{0}\right), \ldots, X\left(k_{q}\right)-X\left(k_{0}\right)\right\}$.

By the monotone convergence theorem, Eq. (2.2) holds for all $f_{-p}, \ldots, f_{q}$ and the proposition follows.

Indeed, the above argument has the following immediate but important consequence. 
Corollary 2.1. For all $A \in \Pi$, every $n, m \in \mathbb{N}_{0}^{N}$ with $n \preccurlyeq(A) m$, and for all $f: \mathbb{Z}^{d} \mapsto \mathbb{R}_{+}$,

$$
\mathbb{E}_{\mathbb{Q}}\left\{f \circ X(m) \mid \mathcal{F}^{A}(n)\right\}=\mathbb{E}\{f[X(m)-X(n)+\beta]\},
$$

$\mathbb{Q}$-almost surely on $\{X(n)=\beta\}$.

\section{Preliminaries on Unimodal Walks}

In this section, we present a sufficient condition for an additive random walk to be weakly unimodal. We also prove a technical result that will be useful in our proof of Theorem 1.2.

It is easy to verify directly that for $N=1$, any simple (nearest neighbor with equal probabilities) random walk on $\mathbb{Z}^{d}$ is weakly unimodal with $\kappa=1$. This can also be derived from the following lemma.

Lemma 3.1. Let $Y$ be a random variable on $\mathbb{Z}^{d}$. If $\mathbb{E}\left\{e^{i \xi \cdot Y}\right\} \geqslant 0$,

$$
\sup _{a \in \mathbb{Z}^{d}} \mathbb{P}\{Y=a\} \leqslant \mathbb{P}\{Y=0\} .
$$

Proof. By Fourier's inversion formula, for all $g \in \mathbb{Z}^{d}$,

$$
\mathbb{P}\{Y=g\}=(2 \pi)^{-d} \int_{[-\pi, \pi]^{d}} e^{-i g \cdot \xi} \mathbb{E}\left\{e^{i \xi \cdot Y}\right\} d \xi
$$

and the result follows immediately.

Example. There are many random variables in $\mathbb{Z}$ that have a positive characteristic function. Here is one construction: consider a sequence $\left\{p_{k}\right\}$ of non-negative numbers such that $p_{0}+\sum_{k=1}^{\infty} p_{k}=1$ and $\sum_{k=1}^{\infty} p_{k} \leqslant p_{0}$ and define $Y$ be a random variable with

$$
\mathbb{P}\{Y=0\}=p_{0}, \quad \mathbb{P}\{Y=k\}=\mathbb{P}\{Y=-k\}=p_{k} / 2 \quad(k \geqslant 1) .
$$

Then, it is clear that $\mathbb{E}\left\{e^{i \xi \cdot Y}\right\} \geqslant 0$. Now, consider $X_{1}, \cdots, X_{N}-N$ independent random walks on $\mathbb{Z}^{d}$-and denote the characteristic function of $X_{j}(1)$ by $\Psi_{j}(j=1, \cdots, N)$, respectively. Then, for any $n \in \mathbb{N}_{0}^{N}, \mathbb{E}[\exp \{i \xi$. $X(n)\}]=\prod_{j=1}^{N}\left[\Psi_{j}(\xi)\right]^{n_{j}}$. There are many possible choices for $N$ and $\Psi_{j}(j=$ $1, \ldots, N)$ such that the above characteristic function is non-negative for all $n \in \mathbb{N}_{0}^{N}$, or more generally $\mathbb{E}[\exp \{i \xi \cdot X(n)\}] \geqslant 0$ for all $n \in \mathbb{N}_{0}^{N}$ with 
$\mathbb{P}\{X(n)=0\}>0$. It follows from Lemma 3.1 that for such choices the corresponding additive random walks are weakly unimodal with $\kappa=1$. In particular, this is the case for the simple random walk in $\mathbb{Z}^{d}$.

Next, we use the order structure on $\mathbb{N}_{0}^{N}$ described in the Introduction: for all $m, n \in \mathbb{N}_{0}^{N}, m \preccurlyeq n$ if and only if $m_{\ell} \leqslant n_{\ell}$, for all $\ell=1, \ldots, N$. We also recall the definitions of $m \curlywedge n$ and $\Psi$.

Lemma 3.2 (Weak Monotonicity). Let $X$ be a weakly unimodal $N$ parameter additive random walk. For $m, n \in \mathbb{N}_{0}^{N}$, if $m \preccurlyeq n$ and $\mathbb{P}\{X(m)=$ $0\}>0$, then $\Psi(n) \leqslant \kappa \Psi(m)$.

Proof. Since $m \preccurlyeq n, X(m)$ and $X(n)-X(m)$ are independent. Hence,

$$
\begin{aligned}
\Psi(n) & =\mathbb{P}\{X(m)+(X(n)-X(m))=0\} \\
& \leqslant \sup _{a \in \mathbb{Z}^{d}} \mathbb{P}\{X(m)+a=0\} \leqslant \kappa \Psi(m),
\end{aligned}
$$

by weak unimodality. This proves the lemma.

Lemma 3.3. Let $X$ be a symmetric and weakly unimodal $N$-parameter additive random walk. For all $m, n \in \mathbb{N}_{0}^{N}$,

$$
\mathbb{P}\{X(m)=0, X(n)=0\} \leqslant \kappa \Psi(m \curlywedge n) \Psi(m-n) .
$$

Proof. If $\mathbb{P}\{X(m)=0, X(n)=0\}=0$, then there is nothing to prove. So, we may assume that this probability is positive. Recall that $n \curlywedge m$ denotes the point in $\mathbb{N}_{0}^{N}$ whose $j$ th coordinate is $n_{j} \wedge m_{j}$ (the minimum). For all $n, m \in \mathbb{N}_{0}^{N}$, let $Z_{1}=X(n)-X(n \curlywedge m)$ and $Z_{2}=X(m)-X(n \curlywedge m)$. Then,

$$
\begin{aligned}
& \mathbb{P}\{X(n)=0, X(m)=0\} \\
& \quad=\mathbb{P}\left\{X(n \curlywedge m)+Z_{1}=0, X(n \curlywedge m)+Z_{2}=0\right\} \\
& \quad \leqslant \mathbb{P}\left\{X(n \curlywedge m)+Z_{2}=0, X(n)-X(m)=0\right\} .
\end{aligned}
$$

Since $X(n \curlywedge m), Z_{1}$ and $Z_{2}$ are mutually independent, the above is bounded above by

$$
\begin{aligned}
& \sup _{a \in \mathbb{Z}^{d}} \mathbb{P}\{X(n \curlywedge m)=a\} \cdot \mathbb{P}\{X(n)-X(m)=0\} \\
& \leqslant \kappa \mathbb{P}\{X(n \curlywedge m)=0\} \cdot \mathbb{P}\{X(n)-X(m)=0\} \\
&=\kappa \Psi(n \curlywedge m) \cdot \mathbb{P}\{X(n)-X(m)=0\} .
\end{aligned}
$$

We have used weak unimodality in the above. By symmetry and independence, one can also directly check that $\mathbb{P}\{X(n)-X(m)=0\}=\Psi(n-m)$, and our proof is complete. 


\section{Proof of Theorem 1.1}

We begin by stating a multiparameter martingale inequality. When the underlying measure is a probability measure, this is due to R. Cairoli; cf. $[3,18]$. When the underlying measure is our infinite but $\sigma$-finite measure $\mathbb{Q}$, a proof can be set forth by following through the standard proof of the probability case carefully. In a one-parameter, discrete setting, the details can be found in [8, Eq. (40.2), p. 34]. One generalizes this development to our present multiparameter setting by applying one-parameter arguments one parameter at a time; this idea is due to R. Cairoli and is well explained in $[18]$.

Lemma 4.1 (Cairoli's (2,2) Maximal Inequality). For $A \in \Pi$ and for any bounded random variable $Y$, define the $N$-parameter $\mathcal{F}^{A}$-martingale $M(m)=\mathbb{E}_{\mathbb{Q}}\left\{Y \mid \mathcal{F}^{A}(m)\right\},\left(m \in \mathbb{N}_{0}^{N}\right)$. Then,

$$
\mathbb{E}_{\mathbb{Q}}\left\{\sup _{m \in \mathbb{N}_{0}^{N}} M(m)^{2}\right\} \leqslant 4^{N} \mathbb{E}_{\mathbb{Q}}\left\{Y^{2}\right\}
$$

Define

$$
J_{\mu}(a)=\sum_{n} \mathbf{1}_{\{X(n)=a\}} \mu(\{n\}), \quad a \in \mathbb{Z}^{d}, \mu \in \mathcal{P}\left(\mathbb{N}_{0}^{N}\right) .
$$

As an immediate consequence of Corollary 2.1, we obtain

Lemma 4.2. For all $a \in \mathbb{Z}^{d}$ and $\mu \in \mathcal{P}\left(\mathbb{N}_{0}^{N}\right)$,

$$
\begin{aligned}
\mathbb{E}_{\mathbb{Q}}\left\{J_{\mu}(a)\right\} & =1 \\
\mathbb{E}_{\mathbb{Q}}\left\{\left|J_{\mu}(a)\right|^{2}\right\} & =\mathcal{E}_{\Phi}(\mu) .
\end{aligned}
$$

We are ready to verify the easier half of Theorem 1.1.

Proof of Theorem 1.1: Lower Bound. By the Paley-Zygmund inequality, for all $a \in \mathbb{Z}^{d}$ and all $\mu \in \mathcal{P}(E)$,

$$
\mathbb{Q}\left\{J_{\mu}(a)>0\right\} \geqslant \frac{\left[\mathbb{E}_{\mathbb{Q}}\left\{J_{\mu}(a)\right\}\right]^{2}}{\mathbb{E}_{\mathbb{Q}}\left\{\left|J_{\mu}(a)\right|^{2}\right\}} ;
$$

see [11] for a proof in the probability case. The standard argument for this works to prove the above formulation for $\mathbb{Q}$, as well. Applying Lemma 4.2 and optimizing over $\mu \in \mathcal{P}(E)$, we can deduce that

$$
\mathbb{Q}\{\exists n \in E: X(n)=a\} \geqslant \mathcal{C}_{\Phi}(E) .
$$


On the other hand,

$$
\begin{aligned}
\mathbb{Q}\{\exists n \in E: X(n)=a\} & =\sum_{x \in \mathbb{Z}^{d}} \mathbb{P}\{\exists n \in E: X(n)+x=a\} \\
& =\mathbb{E}\{\# X(E)\}
\end{aligned}
$$

This completes our proof of the lower bound in Theorem 1.1.

In order to prove the upper bound, we need one technical estimate that involves martingale estimates. Recalling Eq. (4.1), we define for all $\mu \in$ $\mathcal{P}(E), A \in \Pi$ and for every $a \in \mathbb{Z}^{d}$,

$$
M_{\mu}^{a, A}(n)=\mathbb{E}_{\mathbb{Q}}\left\{J_{\mu}(a) \mid \mathcal{F}^{A}(n)\right\}, \quad n \in \mathbb{N}_{0}^{N} .
$$

Lemma 4.3. For all $\mu \in \mathcal{P}(E), A \in \Pi, a \in \mathbb{Z}^{d}$ and for all $n \in \mathbb{N}_{0}^{N}$,

$$
M_{\mu}^{a, A}(n) \geqslant \sum_{\ell \succcurlyeq(A)} \Phi(\ell, n) \mu(\{\ell\}) \cdot \mathbf{1}_{\{X(n)=a\}},
$$

Q-almost surely.

Proof. Combining Eq.'s (4.1) and (4.3), we see that

$$
M_{\mu}^{a, A}(n) \geqslant \sum_{\ell \succcurlyeq(A)} \mathbb{Q}\left\{X(\ell)=a \mid \mathcal{F}^{A}(n)\right\} \mu(\{\ell\}) \cdot \mathbf{1}_{\{X(n)=a\}},
$$

$\mathbb{Q}$-almost surely. By Corollary 2.1, $\mathbb{Q}$-almost surely on $\{X(n)=a\}$,

$$
\mathbb{Q}\left\{X(\ell)=a \mid \mathcal{F}^{A}(n)\right\}=\Phi(\ell, n) .
$$

This completes our proof.

We can now proceed with our

Proof of Theorem 1.1: Upper Bound. By standard Choquet capacity arguments, we can assume that $E$ is a finite set. Let $\tau$ denote any measurable selection of $n \in E$, such that $X(\tau)=a$. If $a \notin X(E)$, we define $\tau \equiv+\infty$. Squaring the expression in Lemma 4.3 and taking suprema over all $n \in \mathbb{N}_{0}$, we see that for all $a \in \mathbb{Z}^{d}, A \in \Pi$ and for all $\mu \in \mathcal{P}(E)$,

$$
\sup _{n \in \mathbb{N}_{0}^{N}}\left|M_{\mu}^{a, A}(n)\right|^{2} \geqslant\left[\sum_{\ell \succcurlyeq(A) \tau} \Phi(\ell, \tau) \mu(\{\ell\})\right]^{2} \cdot \mathbf{1}_{\{\exists n \in E: X(n)=a\}}
$$


$\mathbb{Q}$-almost surely. Since $\sum_{A \in \Pi}\left|x_{A}\right|^{2} \geqslant 2^{-N}\left|\sum_{A \in \Pi} x_{A}\right|^{2}$ for every sequence $\left\{x_{A} ; A \in \Pi\right\}$, we can add over all $A \in \Pi$ and see that for any $\mu \in \mathcal{P}(E)$,

$$
\sum_{A \in \Pi} \sup _{n \in \mathbb{N}_{0}^{N}}\left|M_{\mu}^{a, A}(n)\right|^{2} \geqslant 2^{-N}\left[\sum_{\ell} \Phi(\ell, \tau) \mu(\{\ell\})\right]^{2} \cdot \mathbf{1}_{\{\exists n \in E: X(n)=a\}},
$$

$\mathbb{Q}$-almost surely. Now, we choose a special $\mu$ by defining

$$
\mu(\{\bullet\})=\mathbb{Q}\{\tau=\bullet \mid \tau \neq+\infty\} .
$$

Since $E$ is assumed to be finite, by Eq. (4.2),

$$
1 \leq \mathbb{Q}\{\tau \neq \infty\}=\mathbb{E}\{\# X(E)\} \leqslant \# E<\infty .
$$

Thus, $\mu \in \mathcal{P}(E)$ and by Eq. (4.4), applied to this specific choice of $\mu$,

$$
\begin{aligned}
\sum_{A \in \Pi} & \mathbb{E}_{\mathbb{Q}}\left\{\sup _{n \in \mathbb{N}_{0}^{N}}\left|M_{\mu}^{a, A}(n)\right|^{2}\right\} \\
& \geqslant 2^{-N} \sum_{n}\left[\sum_{\ell} \Phi(\ell, n) \mu(\{\ell\})\right]^{2} \mu(\{n\}) \cdot \mathbb{Q}\{\exists n \in E: X(n)=a\} \\
& \geqslant 2^{-N}\left[\mathcal{E}_{\Phi}(\mu)\right]^{2} \cdot \mathbb{E}\{\# X(E)\} .
\end{aligned}
$$

We have used Eq. (4.2) in the last step, together with the Cauchy-Schwarz inequality. On the other hand, by Lemmas 4.1 and 4.2 , the left hand side is bounded above by

$$
4^{N} \sum_{A \in \Pi} \mathcal{E}_{\Phi}(\mu)=8^{N} \mathcal{E}_{\Phi}(\mu)
$$

We have shown the existence of some $\mu \in \mathcal{P}(E)$, such that

$$
\mathbb{E}\{\# X(E)\} \cdot\left[\mathcal{E}_{\Phi}(\mu)\right]^{2} \leqslant 16^{N} \mathcal{E}_{\Phi}(\mu)
$$

Since $\Phi(n, n)=1, \mathcal{E}_{\Phi}(\mu) \geqslant \sum_{n \in E}|\mu(\{n\})|^{2}>0$. Thus, we can deduce the requisite upper bound by dividing each side of $(4.5)$ by $\mathcal{E}_{\Phi}(\mu)$. This completes our proof.

Remark 4.1. As mentioned after the statement of Theorem 1.1, in the $N=1$ case, constant 16 in (1.4) can be improved to 2 . We now give the requisite argument. The special feature of this 1-parameter setting is that the parameter set $\mathbb{N}_{0}$ is totally ordered by the linear order $\preccurlyeq$ which is now the same as the usual $\leqslant$. Thus, we need only consider the latter 
partial order. We recall that for all $m \in \mathbb{N}_{0}, \mathcal{F}_{m}$ is the $\sigma$-algebra generated by $X(n)$, for all $0 \leqslant n \leqslant m$. For each $\mu \in \mathcal{P}\left(\mathbb{N}_{0}\right)$, consider the martingale $M=\left\{M_{\mu}^{a}(n) ; n \in \mathbb{N}_{0}\right\}$ defined by

$$
M_{\mu}^{a}(n)=\mathbb{E}_{\mathbb{Q}}\left\{J_{\mu}(a) \mid \mathcal{F}_{n}\right\}, \quad n \in \mathbb{N}_{0}
$$

Similar to Lemma 4.3, we obtain that for all $\mu \in \mathcal{P}\left(\mathbb{N}_{0}\right)$,

$$
M_{\mu}^{a}(n) \geqslant \sum_{\ell \geqslant n} \Phi(\ell, n) \mu(\{\ell\}) \cdot \mathbf{1}_{\{X(n)=a\}} .
$$

This holds $\mathbb{Q}$-almost surely for all $n \in \mathbb{N}_{0}$. Define $\tau$ to be the first $n \in \mathbb{N}_{0}$ such that $X(n)=a$, with the usual stipulation that inf $\varnothing=+\infty$. Then, $\tau$ is a stopping time with respect to the filtration $\mathcal{F}=\left\{\mathcal{F}_{n} ; n \in \mathbb{N}_{0}\right\}$. By the optional stopping theorem for $\mathbb{E}_{\mathbb{Q}}$, which is standard to verify, we have

$$
\begin{aligned}
& \mathbb{E}_{\mathbb{Q}}\left\{M_{\mu}^{a}(\tau) \mathbf{1}_{(\tau<+\infty)}\right\} \leqslant \lim _{k \rightarrow \infty} \mathbb{E}_{\mathbb{Q}}\left\{M_{\mu}^{a}(\tau \wedge k)\right\} \\
& =\mathbb{E}_{\mathbb{Q}}\left\{M_{0}^{a}\right\}=1 .
\end{aligned}
$$

Applying (4.6) to $n=\tau$ and taking expectations with respect to $\mathbb{Q}$, we obtain

$$
1 \geqslant \mathbb{E}_{\mathbb{Q}}\left\{\sum_{n \geqslant \tau} \Phi(n, \tau) \mu(\{\ell\}) \cdot \mathbf{1}_{\{\tau<+\infty\}}\right\} .
$$

As this holds for all $\mu \in \mathcal{P}\left(\mathbb{N}_{0}\right)$, we can apply it to

$$
\mu(\bullet)=\mathbb{Q}\{\tau \in \bullet \mid \tau<+\infty\}
$$

and see that

$$
\mathbb{Q}\{\tau<+\infty\} \leqslant\left[\sum_{\ell \geqslant m} \Phi(\ell, m) \mu(\{\ell\}) \mu(\{m\})\right]^{-1} .
$$

Since the above double sum is clearly bounded below by $\frac{1}{2} \varepsilon_{\Phi}(\mu)$, we obtain the upper bound of Theorem 1.1 with the better constant of 2 , in case $N=1$. This proves the assertion of Remark 1.1.

\section{$5 \quad$ Proof of Theorem 1.2}

We are ready to prove Theorem 1.2. In contrast to our presentation of $\S 4$, this time, we begin with the harder half, since we have already done most of the preliminary work. 
Proof of Theorem 1.2: Upper Bound. The upper bound follows readily from the upper bound given in Theorem 1.1. Indeed, for any $a \in \mathbb{Z}^{d}$,

$$
\begin{aligned}
& \mathbb{P}\{\exists n \in E: X(n)=a\} \\
& \quad=\sum_{x \in \mathbb{Z}^{d}} \mathbb{P}\left\{X\left(\delta_{E}\right)=x\right\} \cdot \mathbb{P}\left\{\exists n \in E: X(n)-X\left(\delta_{E}\right)=a-x\right\} \\
& \quad \leqslant \Psi\left(\delta_{E}\right) \kappa \cdot \sum_{x \in \mathbb{Z}^{d}} \mathbb{P}\left\{\exists n \in E: X(n)-X\left(\delta_{E}\right)=a-x\right\} .
\end{aligned}
$$

We have used weak unimodality in the penultimate line. By Theorem 1.1,

$$
\mathbb{P}\{\exists n \in E: X(n)=a\} \leqslant 16^{N} \kappa \Psi\left(\delta_{E}\right) \cdot \mathcal{C}_{\Psi}\left(E \ominus \delta_{E}\right),
$$

where $E \ominus x=\{n-x: n \in E\}$. It is clear from Eq. (1.5), that $F \mapsto \mathcal{C}_{\psi}(F)$ is translation invariant. Thus, $\mathcal{C}_{\Psi}\left(E \ominus \delta_{E}\right)=\mathcal{C}_{\Psi}(E)$ and the upper bound is proved.

The 'lower bound version' of the above argument is not sufficiently sharp. Instead, we adapt the arguments that lead to the lower bound in Theorem 1.1 .

Proof of Theorem 1.2: Lower Bound. If $\Psi\left(\Delta_{E}\right)=0$, there is nothing to prove. Hence, we may assume, with no loss in generality, that $\Psi\left(\Delta_{E}\right)>0$. Using the notation Eq. (4.1) with $\mu \in \mathcal{P}(E)$, let $J_{\mu}=J_{\mu}(0)$ for brevity. Then, by weak unimodality, weak monotonicity (Lemma 3.2) and after a few lines of calculations, we obtain the following.

$$
\begin{aligned}
\mathbb{E}\left\{J_{\mu}\right\} & \geqslant \frac{1}{\kappa} \Psi\left(\Delta_{E}\right) \\
\mathbb{E}\left\{\left|J_{\mu}\right|^{2}\right\} & \leqslant \kappa \Psi\left(\delta_{E}\right) \cdot \mathcal{E}_{\Psi}(\mu) .
\end{aligned}
$$

By the Paley-Zygmund inequality and after optimizing over all $\mu \in \mathcal{P}(E)$, we obtain

$$
\mathbb{P}\{\exists n \in E: X(n)=0\} \geqslant \frac{\Psi^{2}\left(\Delta_{E}\right)}{\kappa^{3} \Psi\left(\delta_{E}\right)} \cdot \mathcal{C}_{\Psi}(E) .
$$

This completes our argument.

\section{Proof of Theorems 1.3 and 1.4}

In this section, we prove Theorem 1.3 and 1.4. 
Proof of Theorem 1.3. Since the event $\{\omega: \# \mathcal{L}=+\infty\}$ is a tail event for all of $X_{1}, \ldots, X_{N}$. The first two assertions of Theorem 1.3 are equivalent. By Fubini's theorem, if assertion 3 does not hold, then $\mathbb{E}\{\# \mathcal{L}\}<\infty$, which implies that 0 is a.s. not recurrent. It suffices to prove the converse. That is,

$$
\mathbb{E}\{\# \mathcal{L}\}=+\infty \Longrightarrow \mathbb{P}\{\# \mathcal{L}=+\infty\}>0
$$

In order to prove this, we first note that by Lemma 3.3,

$$
\begin{aligned}
\mathbb{E}\left\{|\mathcal{L}(n)|^{2}\right\} & \left.\leqslant \sum_{i, j \in[0, n]^{N}} \sum_{\{} \mathbb{P}(i)=X(j)=0\right\} \\
& \leqslant \kappa \sum_{i, j \in[0, n]^{N}} \sum_{(i \curlywedge j) \Psi(i-j)} \\
& =\kappa \sum_{i, j \in[0, n]^{N}} \sum_{(i \curlywedge j) \Psi(i \curlyvee j-i \curlywedge j) .}
\end{aligned}
$$

Thus, we can rearrange the sum to see that $\mathbb{E}\left\{|\mathcal{L}(n)|^{2}\right\}$ is bounded above by

$$
\begin{aligned}
\kappa \sum_{\substack{p, q \in[0, n]^{N}: \\
p \succcurlyeq q}} \Psi(q) \Psi(p-q) \cdot & \#\left\{i, j \in[0, n]^{N}: p=i \curlyvee j, q=i \curlywedge j\right\} \\
& \leqslant 2^{N} \kappa \sum_{\substack{p, q \in[0, n]^{N}: \\
p \succcurlyeq q}} \Psi(q) \Psi(p-q) \\
& \leqslant 2^{N} \kappa\left\{\sum_{p \in[0, n]^{N}} \Psi(p)\right\}^{2} .
\end{aligned}
$$

This proves that $\mathbb{E}\left\{|\mathcal{L}(n)|^{2}\right\} \leqslant \kappa 2^{N}[\mathbb{E}\{\mathcal{L}(n)\}]^{2}$. Consequently, it follows from the Paley-Zygmund inequality that

$$
\begin{aligned}
\mathbb{P}\left\{\mathcal{L}(n) \geqslant \frac{1}{2} \mathbb{E}[\mathcal{L}(n)]\right\} & \geqslant \frac{1}{4} \frac{(\mathbb{E}\{\mathcal{L}(n)\})^{2}}{\mathbb{E}\left\{|\mathcal{L}(n)|^{2}\right\}} \\
& \geqslant \frac{1}{\kappa 2^{N+3}}
\end{aligned}
$$

see $\left[11\right.$, p.8]. On the other hand, whenever $\mathbb{E}\{\# \mathcal{L}\}=+\infty, \lim _{n} \mathbb{E}\{\mathcal{L}(n)\}=$ $\infty$. Since $\mathcal{L}(n) \leqslant \# \mathcal{L}$,

$$
\mathbb{P}\{\mathcal{L}=+\infty\} \geqslant \liminf _{n \rightarrow \infty} \mathbb{P}\left\{\mathcal{L}(n) \geqslant \frac{1}{2} \mathbb{E}[\mathcal{L}(n)]\right\} \geqslant \kappa^{-1} 2^{-N-3}
$$

We have verified Eq. (6.1). 
Proof of Theorem 1.4. We start with our proof of the upper bound in (1.8). For any $\varepsilon>0$, by Eq. (1.7),

$$
\mathbb{E}\{\mathcal{L}(n)\}=\sum_{k \in[0, n]^{N}} \Phi(k) \leqslant K n^{-(\bar{\gamma}+\varepsilon)} \text { for all } n \in \mathbb{N}_{0}^{N}
$$

For every integer $\ell \geqslant 1$, we take $n_{\ell}=2^{\ell}$. Then, by (6.3) and the Chebyshev's inequality we obtain

$$
\mathbb{P}\left\{\mathcal{L}\left(n_{\ell}\right)>2^{-\ell(\bar{\gamma}+2 \varepsilon)}\right\} \leqslant 2^{-\varepsilon \ell} .
$$

The Borel-Cantelli lemma implies that almost surely for all integers $\ell$ that are large enough,

$$
\mathcal{L}\left(n_{\ell}\right) \leqslant 2^{-\ell(\bar{\gamma}+2 \varepsilon)} .
$$

If $n$ is large and if $\ell$ satisfies $2^{\ell} \leqslant n<2^{\ell+1}$, Eq. (6.5) implies that

$$
\mathcal{L}(n) \leqslant \mathcal{L}\left(n_{\ell+1}\right) \leqslant 2^{\bar{\gamma}+2 \varepsilon} n^{\bar{\gamma}+2 \varepsilon} .
$$

Therefore,

$$
\limsup _{n \rightarrow \infty} \frac{\log \mathcal{L}(n)}{\log n} \leqslant \bar{\gamma}+2 \varepsilon . \quad \text { a.s. }
$$

Since $\varepsilon>0$ is arbitrary, this proves the upper bound in (1.8). In order to prove the corresponding lower bound, we note that by Eq. (6.2),

$$
\mathbb{P}\left\{\mathcal{L}(n) \geqslant \frac{1}{2} \mathbb{E}[\mathcal{L}(n)] \text { infinitely often }\right\} \geqslant \frac{1}{\kappa 2^{N+3}} .
$$

By Eq. (1.7), for any $\varepsilon>0$ and all $n \geqslant 1$, there exists a finite and positive constant $K$, such that $\mathbb{E}[\mathcal{L}(n)] \geqslant K^{-1} n \underline{\underline{\gamma}}^{-\varepsilon}$ for all $n$ large enough. This and Eq. (6.6), together imply that

$$
\limsup _{n \rightarrow \infty} \frac{\log \mathcal{L}(n)}{\log n} \geqslant \underline{\gamma}-\varepsilon,
$$

with positive probability. We obtain the asserted lower bound in Theorem 1.4 by making an appeal to the 0-1 law of Hewitt and Savage.

Let us conclude this section by studying a class of additive random walks that have nice, regular behavior. Namely, we assume that $X_{1}, \ldots, X_{N}$ are all independent random walks on $\mathbb{Z}^{d}$ and they all have the same law. Furthermore, we assume the existence of $\alpha>0$, such that for all $n \geqslant 1$,

$$
\mathbb{P}\left\{X_{1}(n)=0\right\} \asymp n^{-\alpha},
$$


where $a(x) \asymp b(x)$ means there exists a finite $C>1$, such that for all $x$ under consideration,

$$
C^{-1} \leqslant \frac{a(x)}{b(x)} \leqslant C
$$

We note that, in this case, for all $n \in \mathbb{Z}^{N}$,

$$
\Psi(n) \asymp\{|n|+1\}^{-\alpha},
$$

where $|\bullet|$ denotes any of the Euclidean norms on $\mathbb{R}^{N}$. It is easy to verify that, in the present setting, $\gamma=\bar{\gamma}=(N-\alpha)^{+}$. It follows from Theorems 1.3 and 1.4 that $X$ is recurrent if and only if $N>\alpha$. Moreover, with probability one,

$$
\limsup _{n \rightarrow \infty} \frac{\log \mathcal{L}(n)}{\log n}=(N-\alpha)^{+} .
$$

\section{REFERENCES}

[1] M. Barlow and S. J. Taylor (1989), Fractal dimensions of sets in discrete spaces, J. Phys. A 22, 2621-2626.

[2] M. Barlow and S. J. Taylor (1992), Defining fractal subsets of $\mathbb{Z}^{d}$, Proc. London Math. Soc. 64(3), 125-152.

[3] R. Cairoli (1968), Une inegalité pour martingales à indices multiples et ses applications, Séminaire de Probabilités IV, 1-27.

[4] R. C. Dalang and T. S. Mountford (1997), Points of increase of the Brownian sheet, Prob. Th. Rel. Fields, 108(1), 1-27.

[5] R. C. Dalang and T. S. Mountford (1996), Nondifferentiability of curves on the Brownian sheet, Ann. Probab., 24(1), 182-195.

[6] R. C. Dalang and J. B. Walsh (1993), Geography of the level sets of the Brownian sheet, Prob. Th. Rel. Fields, 96(2), 153-176.

[7] R. C. Dalang and J. B. Walsh (1993), The structure of a Brownian bubble, Prob. Th. Rel. Fields, 96(4), 475-501.

[8] C. Dellacherie and P.-A. Meyer (1982), Probabilities and Potential, B. Theory of Martingales. North-Holland Publishing Co., Amsterdam. (J. P. Wilson, Trans.) 
[9] S. N. Evans (1987), Multiple points in the sample paths of a Lévy process, Prob. Th. Rel. Fields, 76(3), 359-367.

[10] P. J. Fitzsimmons and T. S. Salisbury (1989), Capacity and energy for multiparameter processes, Ann. Inst. Henri Poincaré: Prob. Stat., 25(3), 325-350.

[11] J.-P. Kahane (1985), Some Random Series of Functions, Cambridge Studies in Advanced Mathematics, Cambridge, U.K.

[12] W. S. Kendall (1980), Contours of Brownian process with severaldimensional times, Z. Wahr. Verw. Geb., 52, 267-276.

[13] D. Khoshnevisan (1999), Brownian sheet images and Bessel-Riesz capacity, Trans. Amer. Math. Soc., 351(7), 2607-2622.

[14] D. Khoshnevisan and Z. Shi (1999), Brownian sheet and capacity, Ann. of Probab., 27(3), 1135-1159.

[15] D. Khoshnevisan and Y. Xiao (1999), Level sets of additive Lévy processes. Preprint.

[16] J.-F. LeGall, J. Rosen and N.-R. Shieh (1989), Multiple points of Lévy processes, Ann. of Probab. 17(2), 503-515.

[17] T. S. Mountford (1993), Estimates of the Hausdorff dimension of the boundary of positive Brownian sheet components, Séminaire de Probabilités, XXVII, 233-255, Lecture Notes in Math., \#1557, Springer, Berlin.

[18] J. B. Walsh (1986), Martingales with a Multidimensional Parameter and Stochastic Integrals in the Plane, Lecture Notes in Math. \#1215, Springer, Berlin, Ed's: G. del Piño and R. Robodello.

Davar Khoshnevisan

Department of Mathematics

University of Utah

155 S 1400 E, JWB 233

Salt Lake City, UT 84112-0090

URL: http://www.math.utah.edu/ davar 
E-mail: davar@math.utah.edu

Yimin Xiao

Department of Mathematics

University of Utah

155 S 1400 E, JWB 233

Salt Lake City, UT 84112-0090

E-mail: xiao@math.utah.edu and

Microsoft Corporation

One Microsoft Way

Redmond, WA 98052 